AperTO - Archivio Istituzionale Open Access dell'Università di Torino

\title{
Extracellular vesicles derived from mesenchymal stem cells induce features of diabetic retinopathy in vitro
}

\section{This is the author's manuscript}

Original Citation:

Availability:

This version is available http://hdl.handle.net/2318/150933

since

Published version:

DOI:10.1007/s00592-014-0672-1

Terms of use:

Open Access

Anyone can freely access the full text of works made available as "Open Access". Works made available under a Creative Commons license can be used according to the terms and conditions of said license. Use of all other works requires consent of the right holder (author or publisher) if not exempted from copyright protection by the applicable law. 


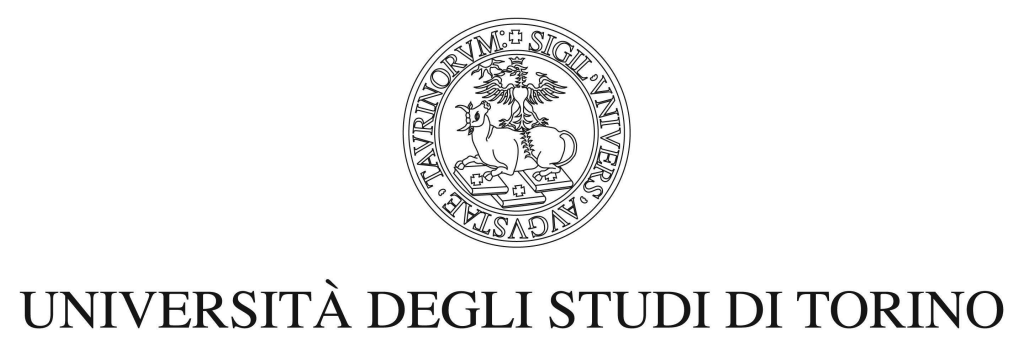

The final publication is available at Springer:

Acta Diabetol. 2014 Dec;51(6):1055-64.

doi: 10.1007/s00592-014-0672-1.

Epub 2014 Nov 6. 


\title{
Extracellular vesicles derived from mesenchymal stem cells induce features of diabetic retinopathy in vitro
}

\author{
Elena Beltramo, Tatiana Lopatina, Elena Berrone, Aurora Mazzeo, Alessandra lavello, \\ Giovanni Camussi and Massimo Porta \\ Department of Medical Sciences, University of Turin, Turin, Italy
}

Running title: Mesenchymal stem cell-derived extracellular vesicles and diabetic retinopathy

\section{Corresponding author:}

Elena Beltramo, MSc PhD, Dept of Medical Sciences, University of Turin, Corso Dogliotti 14, 10126 Torino Italy. Tel+39.011.6708471, Fax+39.011.2368471, elena.beltramo@unito.it

\begin{abstract}
Aims: Loss of pericytes in the early phases of diabetic retinopathy (DR) may disrupt their stable association with endothelial cells (EC), leading to EC proliferation and, eventually, angiogenesis. Extracellular vesicles (EV) are small membrane particles derived from different cells which contain biologically active proteins and RNA and are known to promote phenotypic changes in target cells. In diabetic-like conditions, EV derived from MSC may play a role in vessel destabilization by interfering with the strict interactions between $\mathrm{EC} /$ pericytes and pericyte/extracellular matrix.

Methods: We examined the behaviour of retinal pericytes exposed to EV derived from MSC cultured in physiological and diabetic-like conditions (high glucose and/or hypoxia).

Results: MSC-derived EV are able to enter the pericytes, cause their detachment and migration from the substrate, and increase blood-barrier permeability. Moreover, EV added to EC/pericytes co-cultures in Matrigel promote in vitro angiogenesis. These effects may be mediated by matrix metalloproteinase-2, expressed by both EV and EV-stimulated pericytes, and are exacerbated if MSC are previously cultured in conditions (high glucose and/or hypoxia) mimicking the diabetic microvascular milieu.

Conclusions: We confirm that MSC-derived EV contribute to angiogenesis, showing that they may not only exert a direct stimulus to EC proliferation, but also induce pericyte detachment, thus leaving EC free to proliferate. In addition, we demonstrate a possible link between EV and the early stages of the pathogenesis of DR. Diabetic-like conditions may influence vessel remodelling during angiogenesis through EV paracrine signalling.
\end{abstract}

Keywords: extracellular vesicles, mesenchymal stem cells, pericytes, endothelial cells, diabetic retinopathy, angiogenesis

Abbreviations: DR, diabetic retinopathy; EC, endothelial cells; ECM, extracellular matrix; EV, extracellular vesicles; FCS, fetal calf serum; FITC, fluorescein isothiocyanate; HG, high glucose; HRP, human retinal pericytes; hypo, hypoxia; MMP, matrix metalloproteinases; MSC, mesenchymal stem cells; NG, normal glucose. 


\section{Introduction}

Micro- and macrovascular alterations are key features of the diabetic condition. However, while the retina may be affected by increased revascularization, leading to proliferative diabetic retinopathy (DR), other organs show a marked inhibition of angiogenesis, which in turn leads to peripheral vascular and coronary heart disease (the angiogenic paradox in diabetes) [1].

Pericytes and their interactions with endothelial cells (EC) in the vessel wall have come into focus as central processes in the regulation of vessel formation, stabilization and remodelling [2], thus playing a key role in the pathogenesis of such vascular abnormalities as diabetic microangiopathy (in particular DR), hypertension, stroke, tumour formation and neurodegenerative diseases [3, 4]. If EC are exposed to the complex signals deriving from the blood flow, pericytes receive, and transmit to the endothelium, signals from the surrounding tissues.

Mesenchymal stem cells (MSC) are ubiquitous pluripotent stem cells, whose presence in human eye tissues such as retrocorneal membranes, vitreous, orbital adipose tissue, has been ascertained [5]. A possible contribution of MSC to the physiological and pharmacological repair of tissue damage in several organs has been postulated [5-7]. This may be mediated through transfer of genetic information by extracellular vesicles (EV) [8-11], small membrane particles which include exosomes and microvesicles [12] and represent one of the most promising paracrine signalling pathways. They are released by several cell types in physiological conditions and in response to stress or pathological stimuli [13] and are able to regulate cell migration, proliferation and differentiation, as they contain several biologically active molecules [13-15]. In particular, an important role of $E V$ in the induction of angiogenesis has been suggested [12-15].

EV have been described as potential biomarkers for metabolic diseases, in particular type II diabetes [13]. Circulating EV concentration is increased in diabetic animal models [13] and in subjects with type II diabetes $[16]$ or the metabolic syndrome [17, 18], compared with healthy controls. Monocyte-derived EV have been found increased in patients with diabetic nephropathy and could be markers of microangiopathy [19].

Pericyte survival depends also on their interactions with the extracellular matrix (ECM), whose proteins are susceptible to rapid degradation by metalloproteinases (MMP). Hyperglycaemia-induced upregulation of MMP-2 has been demonstrated in the arterial vasculature in vivo [20] and, in vitro, in several cell types [21, 22] including pericytes [23].

Our working hypothesis is that, in diabetic-like conditions, EV derived from MSC may play a role in vessel destabilization by interfering with the strict EC/pericytes and pericyte/ECM interactions. In particular, EV might stimulate pericyte migration and their loss of contact with EC, thus influencing abnormal angiogenesis.

Hence, this study was aimed at examining the behaviour of retinal pericytes exposed to EV derived from MSC cultured in physiological and diabetic-like conditions (hyperglycaemia and/or hypoxia) and to evaluate the possible role of MMP-2. 


\section{Materials and Methods}

Reagents were purchased by Sigma-Aldrich, unless otherwise stated.

\section{Cell cultures}

Human retinal pericytes (HRP) were immortalized in our laboratory [24]. Human bone marrow and adipose MSC, and human microvascular EC (HMEC) were purchased from Lonza. HRP and MSC were grown in DMEM + 10\%FCS, HMEC in EBM-2 growth medium (Lonza) supplemented with angiogenic factors, according to the instructions.

\section{EV isolation}

MSC were cultured for 8 days in physiological $(5.6 \mathrm{mmol} / \mathrm{L}, \mathrm{NG})$ or high glucose $(28.0 \mathrm{mmol} / \mathrm{L}, \mathrm{HG})$. Hypoxic conditions were obtained by keeping cultures in $5 \% \mathrm{CO}_{2} / 94 \% \mathrm{~N}_{2} / 1 \% \mathrm{O}_{2}$ gas mixture for the last $24 \mathrm{hrs}$ (NG hypo, HG hypo). MSC were serum-deprived 24 hrs prior to $\mathrm{EV}$ isolation.

Cell debris and apoptotic bodies were removed from the supernatants by centrifugation for $30^{\prime}$ at 3,000 and $10,000 \mathrm{~g}$ respectively, then EV were isolated by a further ultracentrifugation at $100,000 \mathrm{~g}$ for $3 \mathrm{hrs}$ at $4^{\circ} \mathrm{C}$. They were used immediately or stored at $-80^{\circ} \mathrm{C}$ in DM EM $+5 \% \mathrm{DMSO}$. No differences in biological activity were observed between fresh and stored EV. EV protein content was quantified by Bradford method, and their size distribution and number using a NanoSight LM10, through the Nanoparticle Tracking Analysis software. For all experiments an EV concentration correspondent to that in MSC supernatants $\left(1 \times 10^{11} \pm\right.$ $1 \times 10^{3} \mathrm{EV} / \mathrm{ml}$, corresponding to $1 \times 10^{5} \pm 1 \times 10^{2} \mathrm{EV} /$ cell) was used.

To investigate EV uptake by HRP, EV were labelled with the red fluorescent aliphatic chromophore PKH26 dye, which intercalates into lipid bilayers. HRP were stained with a FITC-conjugate anti- $\alpha$-smooth muscle actin, and nuclei counterstained with DAPI. Confocal microscopy was performed using a Zeiss microscope, model LSM 5 PASCAL (Jena).

For MMP inhibition studies, EV were pre-incubated with $1 \mu \mathrm{mol} / \mathrm{L}$ batimastat (Abcam) for 15 min prior to addition to HRP.

\section{Cell survival variables}

Cell were counted in Bürker chambers after Trypan blue staining by 2 individual operators. Apoptosis was evaluated by the DeadEnd Colorimetric TUNEL System (Promega). Positive controls were HRP treated with $30 \mathrm{U} / \mathrm{ml}$ RNase-free DNase I (Roche), while negative control cells were incubated without the Terminal Deoxynucleotidyl Transferase. Images were taken under a Leica DM 2000 microscope, equipped with a Leica DFC 320 camera and Leica QWin Plus 2003 digital processing and analysis software.

\section{Migration}

HRP migration was investigated through a Microlmage analysis system (Casti Imaging), by digitally saving images of the same fields at 30 -min intervals over a $12 \mathrm{hr}$ period. Migration tracks were generated by marking the position of the nuclei of individual cells on each image [25]. 


\section{Permeability}

40,000 EC/well were seeded on the inner surface of $0.45-\mu \mathrm{m}$ pore-transwell inserts (Corning) and left to adhere for 24 hrs. 40,000 HRP were subsequently added onto the same insert, as previously described [26]. After another $24 \mathrm{hrs}$, inserts were washed and moved to clean the wells. $600 \mu \mathrm{l}$ DMEM without red phenol and FCS were added to the lower chamber, while $200 \mu \mathrm{l}$ of the same medium supplemented with EV was added into the inserts. After $2 \mathrm{hrs}$, FITC-dextran $(100 \mu \mathrm{g} / \mathrm{ml}$ final concentration) was added into the upper chamber, and fluorescence measured in the lower chamber after another 30', 1, 2, 3, 4 and 24hrs, through a Victor-3 Multilabel Plate Reader (Perkin Elmer).

\section{Vessel-like formation assay}

15,000 HRP and 15,000 EC/well were seeded together onto Matrigel-coated 24-well plates and cultured in serum-deprived DMEM added with EV. Control cultures were obtained seeding 30,000 HRP or 30,000 EC alone in Matrigel-coated wells.

After 24 and $48 \mathrm{hr}$ incubation, phase-contrast images were recorded, and the total length of the network structures measured using the Microlmage analysis system [27] in five random fields and expressed as ratio of control without EV.

\section{Zymography}

Zymography was performed on EV ( $3 \mu \mathrm{g}$ proteins/well) and HRP conditioned media ( $30 \mu \mathrm{l} /$ well), using Ready Zymogram Precast Gels (Biorad) (10\%) copolymerized with $1 \mathrm{mg} / \mathrm{ml}$ gelatine. Samples were mixed with Laemmli's buffer without $\beta$-mercaptoethanol and incubated for 10' at RT. After electrophoresis, gels were

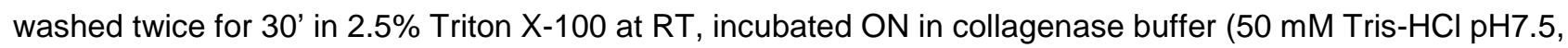
$10 \mathrm{mM} \mathrm{CaCl}_{2}, 150 \mathrm{mM} \mathrm{NaCl}$ ) at $37^{\circ} \mathrm{C}$, and then stained with Cooma ssie brilliant blue. Gelatinolytic activity was visualized as clear bands on a blue background, signal strengths were quantified using a densitometric software (1D Image Analysis System, Kodak).

\section{Statistical analysis}

Results are mean \pm SD of 5 independent experiments, normalized against control (NG cultures without EV). Statistical comparisons among groups were carried out by two-tailed Student's t-test for paired data or Wilcoxon's Signed Ranks test, as appropriate. Results were considered significant for $p \leq 0.05$. SPSS software version 22.0 (IBM) was used for statistical analysis. 


\section{Results}

\section{EV are internalized into human retinal pericytes (HRP)}

EV were collected from the supernatant of bone marrow-derived MSC cultured in the above described conditions (NG, HG, NG hypo, HG hypo), to mimic the diabetic milieu, especially of the retina, where pericytes play a major role in the development of the microvascular disease and hypoxia is a recurrent condition.

NanoSight analysis showed super-imposable concentrations and similar mean size for EV obtained in all conditions (NG: $128 \pm 49 \mathrm{~nm}$, HG: $148 \pm 41 \mathrm{~nm}$, NG hypo: $138 \pm 43 \mathrm{~nm}$, HG hypo: $140 \pm 44 \mathrm{~nm}$ ), while agarose gel electrophoresis demonstrated that EV contain RNA, but not DNA.

EV were able to enter pericytes already after $1 \mathrm{hr}$ incubation and locate inside the cytoplasm, as shown by confocal microscopy, when PKH26-marked EV were added to HRP (Fig.1). No differences in HRP internalization was observed using EV obtained in different conditions.

\section{MSC-derived EV stimulate HRP detachment and migration}

EV were added to sub-confluent HRP cultures and the number of HRP still attached was determined at 0, 2, 4, 6 and $24 \mathrm{hrs}$. We observed a time-dependent detachment of HRP induced by EV obtained in all conditions (Fig. 2a), the major effect occurring after 2 hrs. Detachment was higher when HRP were exposed to EV obtained in diabetic-like conditions. HRP number after 2 hrs exposure to EV obtained in NG (NG-EV) was $31.8 \%$ in comparison with control (HRP not exposed to EV, $p<0.05$ ); HG-EV: $-62.9 \%$ ( $p<0.05$ vs control and NG-EV), NG hypo-EV: $-48.8 \%$ ( $p<0.05$ vs control and NG-EV), HG hypo-EV: $-66.2 \%$ ( $p<0.05$ vs control and NG-EV) (Fig. 2b). These results indicate that EV exposure leads to pericyte detachment from their substrate, and that diabetic-like conditions exacerbate such effect.

Increased HRP motility was observed after addition of EV, particularly those obtained in hypoxia (Fig. 2c). The higher increase was observed after $1 \mathrm{hr}$ EV exposure in all experimental conditions: NG-EV $+37.1 \%$ vs control (NS), HG-EV +43.6\% ( $p<0.05)$, NG hypo-EV +47.0\% $(p<0.05)$, HG hypo-EV $+56.0 \%(p<0.05)$ (Fig. 2d). This suggests that exposure to EV may enhance HRP migration.

To evaluate if these effects were tissue-specific, we checked our findings on HRP detachment using, in addition to EV from bone marrow MSC, EV extracted from adipose MSC and from EC, in physiological conditions (NG). A significant decrease in the number of pericytes exposed to EV from both types of MSC was observed. After 2 hrs, bone marrow MSC-EV: $-23.6 \%$ ( $p<0.005$ vs control), adipose MSC-EV: $-17.4 \%$, $(p<0.005)$, while EV from EC had no effect at all. Interestingly, after $24 \mathrm{hrs}$ HRP treated with HMEC-EV were significantly increased in number $(+5.0 \%$ vs untreated HRP, $p<0.005)$ (Fig. $2 e)$. Hence, we suppose that EV effects on pericyte detachment are common to MSC of different tissue-origin, while EC-derived MSC have an opposite effect, probably due to in vivo tight interactions with pericytes.

\section{EV stimulation does not change survival parameters in HRP}

HRP released in the supernatant, i.e. the cells that had detached after EV stimulation, were collected and seeded in new plates, where they were able to re-attach. The percentage of dead cells, measured by Trypan blue staining, was $<10 \%$. TUNEL assay performed on these new cultures showed no signs of apoptosis (Fig. $3)$, confirming that detached pericytes maintain their viability. 


\section{EV increase blood-barrier permeability}

FITC filtration through our blood-barrier model was augmented by exposure to EV (Fig. 4a), the highest percentage increase occurring after $6 \mathrm{hrs}$ of EV exposure (2 hrs pre-treatment $+4 \mathrm{hrs}$ FITC). Permeability was further increased when co-cultures were exposed to EV obtained in hyperglycaemic and/or hypoxic conditions: NG-EV $+19.8 \%$ ( $p<0.05$ vs control without EV), HG-EV $+33.8 \%$ ( $p<0.005$ vs control, $p<0.05$ vs NG-EV), NG hypo-EV $+25.6 \%$ ( $p<0.05$ vs control, $p=0.000$ vs $N G-E V), H G$ hypo-EV $+35.8 \%$ ( $p<0.05$ vs control and NG-EV) (Fig. 4b). Therefore we hypothesize that EV (especially those in diabetic-like conditions) are able to enhance the retinal blood-barrier permeability.

\section{EV enhance in vitro formation of vessel-like structures by HRP/EC co-cultures}

Addition of EV to HRP/EC co-cultures on Matrigel promoted the formation of vessel-like structures. In control wells, after 48 hrs in serum-free medium, EC and HRP grown separately were simply sub-confluent and no vessel-like structures were present. On the contrary, in wells where EC and HRP had been seeded together, vessel-like structures had formed. EV addition to EC/HRP co-cultures enhanced the number of newly-formed structures in all cases, in particular with EV obtained in hyperglycaemic conditions $(+34.6 \%$ vs control without $E V, p<0.05)$, while hypoxia together with $H G$ had a synergist effect $(+54.3 \%, p<0.05$ vs control and HG alone) (Fig. 5).

\section{EV action on HRP is mediated by MMP-2}

EV from MSC in all experimental conditions contained the active form of MMP-2 (62 kDa) (Fig. 6a), which on the contrary was scarcely expressed by EC-derived EV (Fig. 6b). In addition, after $48 \mathrm{hr}$ EV stimulation, the expression of active MMP-2 in HRP supernatants was significantly increased in comparison with untreated controls (NG-EV: $+51.1 \%, p<0.05$; HG-EV:+48.0, p<0.005; NG hypo-EV: $+75.8 \%, p<0.05$; HG hypo-EV: $+37.9 \%, p<0.05$ ) (Fig. 6c). Consistently with the above results (Fig. 6b), supernatants of HRP treated with EC-derived EV showed very low MMP-2 expression (Fig. 6d).

To check the role of MMP-2, we pre-treated EV with batimastat, a broad-spectrum MMP inhibitor, whose effect on MMP-2 has been described [28], and subsequently exposed HRP to them. HRP number decreased after $2 \mathrm{hr}$ incubation with untreated EV in all conditions, consistently with previous observations described in Fig.2 a-b, while pre-incubation of EV with batimastat completely reverted their effect on pericyte detachment in all conditions, thus confirming MMP involvement in pericyte destabilization (Fig. 6e).

Finally, to check that batimastat does not impede EV internalization into HRP, we exposed HRP to PKH26stained EV obtained in all diabetic-like conditions, with or without pre-treatment with batimastat. We found no difference in EV internalization in all cases. Figure $6 \mathrm{f}-\mathrm{g}$ show sample images of HRP exposed to EV (f) and batimastat pre-treated EV (g). 


\section{Discussion}

Our results show that MSC-derived EV are able to enter the pericytes, causing their detachment from the substrate and migration, possibly contributing to increased blood-barrier permeability in vivo. Moreover, EV stimulate angiogenesis in vitro. These effects are mediated by MMP-2, expressed by both EV and EVstimulated pericytes.

Previous studies had demonstrated that EV derived from MSC of different origin induce angiogenesis, contributing to tissue remodelling after injury $[12,29]$. However, what is a beneficial effect in most tissues and in several diseases, may not be necessarily the same in others. A sort of angiogenesis paradox operates in diabetes: while inappropriate neovascularization may occur in the retina, leading to proliferative $\mathrm{DR}$, in other districts marked inhibition of angiogenesis may lead to peripheral vascular and coronary heart disease [1].

In this work we demonstrate that EV are able to induce angiogenesis not only through direct stimulation of EC growth, but also indirectly, inducing pericyte detachment and migration. This may lead to a lack of control on EC proliferation and thus to angiogenesis. This is confirmed by the finding that addition of EV to $\mathrm{EC} /$ pericytes co-cultures in Matrigel increases the number of newly-formed vessels.

Loss of pericytes, together with thickening of the basement membrane, is considered hallmarks of the early phases in the pathogenesis of DR, with major consequences on vessel remodelling [2, 4, 30]. Pericytes regulate capillary tone and pressure and play a major role in EC proliferation [31]: when their control fails, endothelium enters an angiogenic process, which may lead to proliferative DR [30]. Systemic and local hypertension, hyperglycaemia, advanced glycation end-product (AGE) formation and hypoxia can induce angiogenesis and retinal neovascularisation. Another hallmark of DR is increased vascular permeability, also linked to early loss of pericytes [29]. In this paper, we show increased filtration through EC/pericytes bi-layers exposed to EV.

Consistently with the hypothesized role of EV in the pathogenesis of DR, the above described effects of MSC-derived EV exposure on pericytes and EC/pericytes co-cultures are exacerbated if MSC are previously cultured in conditions (high glucose and/or hypoxia) that mimic the diabetic microvascular milieu.

It is generally acknowledged that hypoxia, a cause of proliferative DR [30], is also a mediator of tumour development and aggressiveness [32], through the induction of growth factors, proteases and cytokines that leads to angiogenesis [33]. Recent data show that EV derived from glioblastoma cells cultured in hypoxic conditions induce angiogenesis ex vivo and in vitro through phenotypic EC modulation, and EC treated with glioblastoma cell-derived EV in hypoxia stimulate pericyte migration [32]. A role for hypoxia and inflammation, but not for high glucose, has been claimed for the altered protein and RNA content of ECreleased EV in stress conditions [34]. Pre-treatment of cultured mesangial cells with high glucose has been shown to increase miR-145 content in mesangial cells-derived EV [35].

However, a link between hyperglycaemic-like conditions and increased angiogenic potential of EV had not been hypothesized so far. In our experimental setting, we show that pericyte detachment from the substrate and permeability through EC/pericyte bi-layers is increased to a greater extent when EV are obtained from 
MSC pre-cultured in high glucose and/or hypoxia conditions, while vessel formation in vitro seems to be increased by hyperglycaemic-like conditions, more than hypoxia.

Pericytes detached from their substrate following EV stimulation maintain their viability, as they were able to re-attach to new culture substrate and did not undergo apoptosis. Since pericytes are necessary for the stabilization of newly-formed vessels during angiogenesis [36], we hypothesize that pericytes detached from old vessels following EV stimulation might be able to migrate to other districts, possibly to stabilize new vessels, consistently with previous observations [37].

We also demonstrate that EV-induced pericyte detachment is specific to EV derived from MSC, as EV extracted from EC supernatants not only showed no effect, but seemed to increase pericyte number. This is confirmed by our finding of a lower content of MMP-2 in EC-EV in comparison with MSC-derived ones, as well as in supernatants of HRP exposed to them, and it is consistent with the fact that in vivo, especially in the retina, pericytes and EC live in tight contact and exert control on each other proliferation [31].

MMP are a class of enzymes which play a crucial role in angiogenesis: they proteolytically cleave several ECM components, determining release of pericytes and loosening of EC/ECM bounds, thus facilitating EC colonization [38]. MMP expression is usually low in physiological conditions, but is strongly up-regulated when ECM remodelling is required, such as in tumour invasion [39]. Hyperglycaemia-induced upregulation of MMP-2 has been demonstrated in several cell types [20-22], including pericytes [23]. Consistently with previous data [12], EV extracted from MSC in our lab strongly express MMP-2, while those extracted from EC scarcely do. Moreover, exposure of pericytes to MSC-derived, but not EC-derived, EV increase MMP-2 expression, and this may explain their detachment from the substrate, when exposed to EV stimulation. The key-role of MMP-2 in our findings is confirmed by the fact that its inhibition by pre-incubating EV in physiological and pathological conditions with the MMP-inhibitor batimastat completely reverted EV effect on pericyte detachment.

In conclusion, we confirm the contribution of MSC-derived EV to angiogenesis, showing that they may not only constitute a direct stimulus to EC proliferation, but also induce pericyte detachment, leaving EC free to proliferate. In addition, we demonstrate, for the first time in our knowledge, a possible link between extracellular vesicles and the early stages of DR. We can therefore hypothesize that diabetic-like conditions may influence vessel stabilization during angiogenesis through EV paracrine signalling. However, studies are needed to further investigate the molecular mechanisms involved in these new findings.

\section{Acknowledgements}

This work was supported by an European Foundation for the Study of Diabetes/Novartis award.

The authors are grateful to dr. Patrizia Dentelli and dr. Maria Chiara Deregibus for technical support and advice.

\section{Conflict of interest}

Elena Beltramo, Tatiana Lopatina, Elena Berrone, Aurora Mazzeo, Alessandra lavello, Giovanni Camussi and Massimo Porta declare that they have no conflict of interest. 


\section{Statement of Human and Animal Rights}

This article does not contain any studies with human or animal subjects performed by the any of the authors.

\section{References}

1. Costa PZ, Soares R (2013) Neovascularization in diabetes and its complications. Unraveling the angiogenic paradox. Life Sci 92:1037-45.

2. Armulik A, Abramsson A, Betsholtz C (2005) Endothelial/pericyte interactions. Circ Res 97:512-23.

3. Raza A, Franklin MJ, Dudek AZ (2010) Pericytes and vessel maturation during tumor angiogenesis and metastasis. Am J Hematol 85:593-98.

4. Beltramo E, Porta M (2013) Pericyte loss in diabetic retinopathy: mechanisms and consequences. Curr Med Chem 20:3218-25.

5. Dhamodaran K, Subramani M, Ponnalagu M, Shetty R, Das D (2014) Ocular stem cells: a status update! Stem Cell Res Ther 5:56.

6. Wollert KC, Drexler H (2005) Mesenchymal stem cells for myocardial infarction: promises and pitfalls. Circulation 112:151-53.

7. Cantley LG (2005) Adult stem cells in the repair of the injured renal tubule. Nat Clin Pract Nephrol 1: 22-32.

8. Bruno S, Grange C, Deregibus MC, Calogero RA, Saviozzi S, Collino F, Morando L, Busca A, Falda M, Bussolati B, et al (2009) Mesenchymal stem cell-derived microvesicles protect against acute tubular injury. J Am Soc Nephrol 20:1053-67.

9. Ratajczak MZ (2011) The emerging role of microvesicles in cellular therapies for organ/tissue regeneration. Nephrol Dial Transplant 26:1453-56.

10. Lopatina T, Kalinina N, Karagyaur M, Stambolsky D, Rubina K, Revischin A, Pavlova G, Parfyonova Y, Tkachuk V (2011) Adipose-derived stem cells stimulate regeneration of peripheral nerves: BDNF secreted by these cells promotes nerve healing and axon growth de novo. PLoS One 6:e17899.

11. Cantaluppi V, Gatti S, Medica D, Figliolini F, Bruno S, Deregibus MC, Sordi A, Biancone L, Tetta C, Camussi $G$ (2012) Microvesicles derived from endothelial progenitor cells protect the kidney from ischemia-reperfusion injury by microRNA-dependent reprogramming of resident renal cells. Kidney Int 82:412-27. 
12. Lopatina T, Bruno S, Tetta C, Kalinina N, Porta M, Camussi G (2014) Platelet-derived growth factor regulates the secretion of extracellular vesicles by adipose mesenchymal stem cells and enhances their angiogenic potential. Cell Commun Signal 12:26.

13. Muller G (2012) Microvesicles/exosomes as potential novel biomarkers of metabolic diseases. Diabetes Metab Syndr Obes 5:247-82.

14. Thery C, Ostrowski M, Segura E (2009) Membrane vesicles as conveyors of immune responses. Nat Rev Immunol 9:581-93.

15. Camussi G, Deregibus MC, Tetta C (2010) Paracrine/endocrine mechanism of stem cells on kidney repair: role of microvesicle-mediated transfer of genetic information. Curr Opin Nephrol Hypertens 19:7-12.

16. Diamant M, Nieuwland R, Pablo RF, Sturk A, Smit JW, Radder JK (2002) Elevated numbers of tissue-factor exposing microparticles correlate with components of the metabolic syndrome in uncomplicated type 2 diabetes mellitus. Circulation 106:2442-47.

17. Agouni A, Lagrue-Lak-Hal AH, Ducluzeau PH, Mostefai HA, Draunet-Busson C, Leftheriotis G, Heymes C, Martinez MC, Andriantsitohaina R (2008) Endothelial dysfunction caused by circulating microparticles from patients with metabolic syndrome. Am J Pathol 173:1210-19.

18. Helal O, Defoort C, Robert S, Marin C, Lesavre N, Lopez-Miranda J, Risérus U, Basu S, Lovegrove $\mathrm{J}$, McMonagle $\mathrm{J}$, et al (2010) Increased levels of microparticles originating from endothelial cells, platelets and erythrocytes in subjects with metabolic syndrome: relationship with oxidative stress. Nutr Metab Cardiovasc Dis 21:665-71.

19. Sabatier F, Darmon P, Hugel B, Combes V, Sanmarco M, Velut JG, Arnoux D, Charpiot P, Freyssinet JM, Oliver C, et al (2002) Type 1 and type 2 diabetic patients display different patterns of cellular microparticles. Diabetes 51:2840-45.

20. Chung AW, Hsiang YN, Matzke LA, McManus BM, van Breemen C, Okon EB (2006) Reduced expression of vascular endothelial growth factor paralleled with the increased angiostatin expression resulting from the upregulated activities of matrix metalloproteinase-2 and -9 in human type 2 diabetic arterial vasculature. Circ Res 99:140-8.

21. Death AK, Fisher EJ, McGrath KCY, Yue DK (2003) High glucose alters matrix metalloproteinase expression in two key vascular cells: Potential impact on atherosclerosis in diabetes. Atherosclerosis 168:263-69. 
22. Hao F, Yu JD (2003) High glucose enhance expression of matrix metalloproteinase-2 in smooth muscle cells. Acta Pharmacol Sin 24:534-38.

23. Tarallo S, Beltramo E, Berrone E, Dentelli P, Porta M (2010) Effects of high glucose and thiamine on the balance between matrix metalloproteinases and their tissue inhibitors in vascular cells. Acta Diabetol 47:105-11.

24. Berrone E, Beltramo E, Buttiglieri S, Tarallo S, Rosso A, Hammes HP, Porta M (2009) Establishment and characterization of a human retinal pericyte line: a novel tool for the study of diabetic retinopathy. Int J Mol Med 23:373-78.

25. Deregibus MC, Buttiglieri S, Russo S, Bussolati B, Camussi G (2003) CD40-dependent activation of phosphatidylinositol 3-kinase/Akt pathway mediates endothelial cell survival and in vitro angiogenesis. J Biol Chem 278:18008-14.

26. Tarallo S, Beltramo E, Berrone E, Porta M (2012) Human pericyte-endothelial cell interactions in coculture models mimicking the diabetic retinal microvascular environment. Acta Diabetol 49 Suppl $1: S 141-51$

27. Bussolati B, Deambrosis I, Russo S, Deregibus MC, Camussi G (2003) Altered angiogenesis and survival in human tumor-derived endothelial cells. FASEB $\mathrm{J}$ 17:1159-61.

28. Brown PD, Giavazzi R (1995) Matrix metalloproteinase inhibitions: a review of the anti-tumour activity of batimastat. Ann Oncol 6:967-974.

29. Bruno S, Camussi G (2013) Role of mesenchymal stem cell-derived microvesicles in tissue repair. Pediatr Nephrol 23:2249-54.

30. Hammes HP (2005) Pericytes and the pathogenesis of diabetic retinopathy. Horm Metab Res 37 Suppl 1:39-43.

31. Hirschi KK, D'Amore PA (1997) Control of angiogenesis by the pericyte: molecular mechanisms and significance. EXS 79:419-28.

32. Kucharzewska P, Christianson HC, Welch JE, Svensson KJ, Fredlund E, Ringnér M, Mörgelin M, Bourseau-Guilmain E, Bengzon J, Belting M (2013) Exosomes reflect the hypoxic status of glioma cells and mediate hypoxia-dependent activation of vascular cells during tumor development. Proc Natl Acad Sci USA 110:7312-17.

33. Liao D, Johnson RS (2007) Hypoxia: A key regulator of angiogenesis in cancer. Cancer Metastasis Rev 26:281-90. 
34. de Jong OG, Verhaar MC, Chen Y, Vader P, Gremmels H, Posthuma G, Schiffelers RM, Gucek M, van Balkom BW (2012) Cellular stress conditions are reflected in the protein and RNA content of endothelial cell-derived exosomes. J Extracell Vesicles 1.

35. Barutta F, Tricarico M, Corbelli A, Annaratone L, Pinach S, Grimaldi S, Bruno G, Cimino D, Taverna D, Deregibus MC, et al (2013) Urinary Exosomal MicroRNAs in Incipient Diabetic Nephropathy. PLoS One 8:e73798.

36. Gerhardt H, Betsholtz C (2003) Endothelial-pericyte interactions in angiogenesis. Cell Tissue Res 314:15-23.

37. Pfister F, Feng Y, vom Hagen F, Hoffmann S, Molema G, Hillebrands JL, Shani M, Deutsch U, Hammes HP (2008) Pericyte migration: a novel mechanism of pericyte loss in experimental diabetic retinopathy. Diabetes 57:2495-502.

38. Rundhaug JE (2005) Matrix metalloproteinases and angiogenesis. J Cell Mol Med 9:267-85.

39. Westermarck J, Kähäri VM (1999) Regulation of matrix metalloproteinase expression in tumor invasion. FASEB J 13:781-92. 


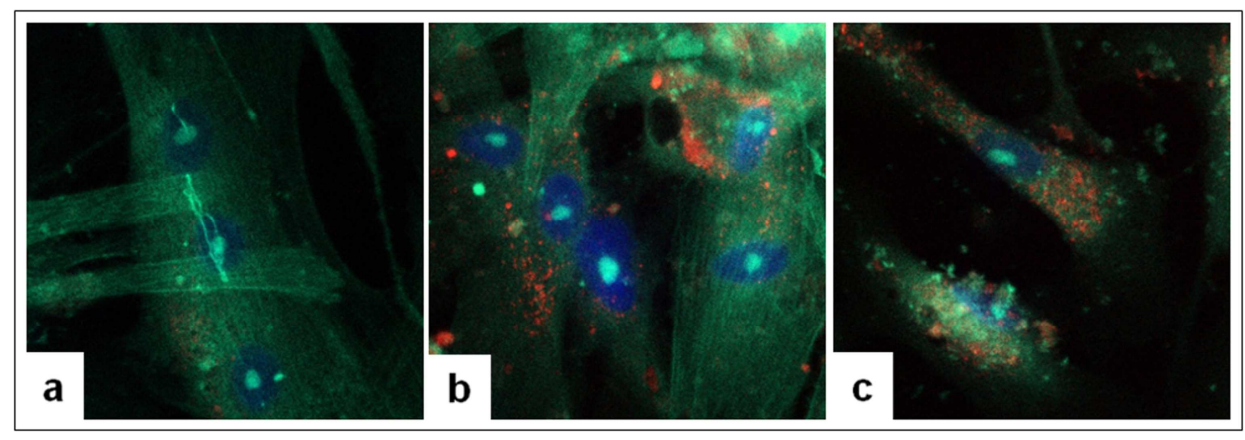

Fig. 1 EV internalize into HRP a) control: anti- $\alpha$-smooth muscle actin and DAPI-stained HRP (green and blue, respectively) without EV; b) HRP incubated for 2 and c) 4 hrs with PKH26-stained EV (red)

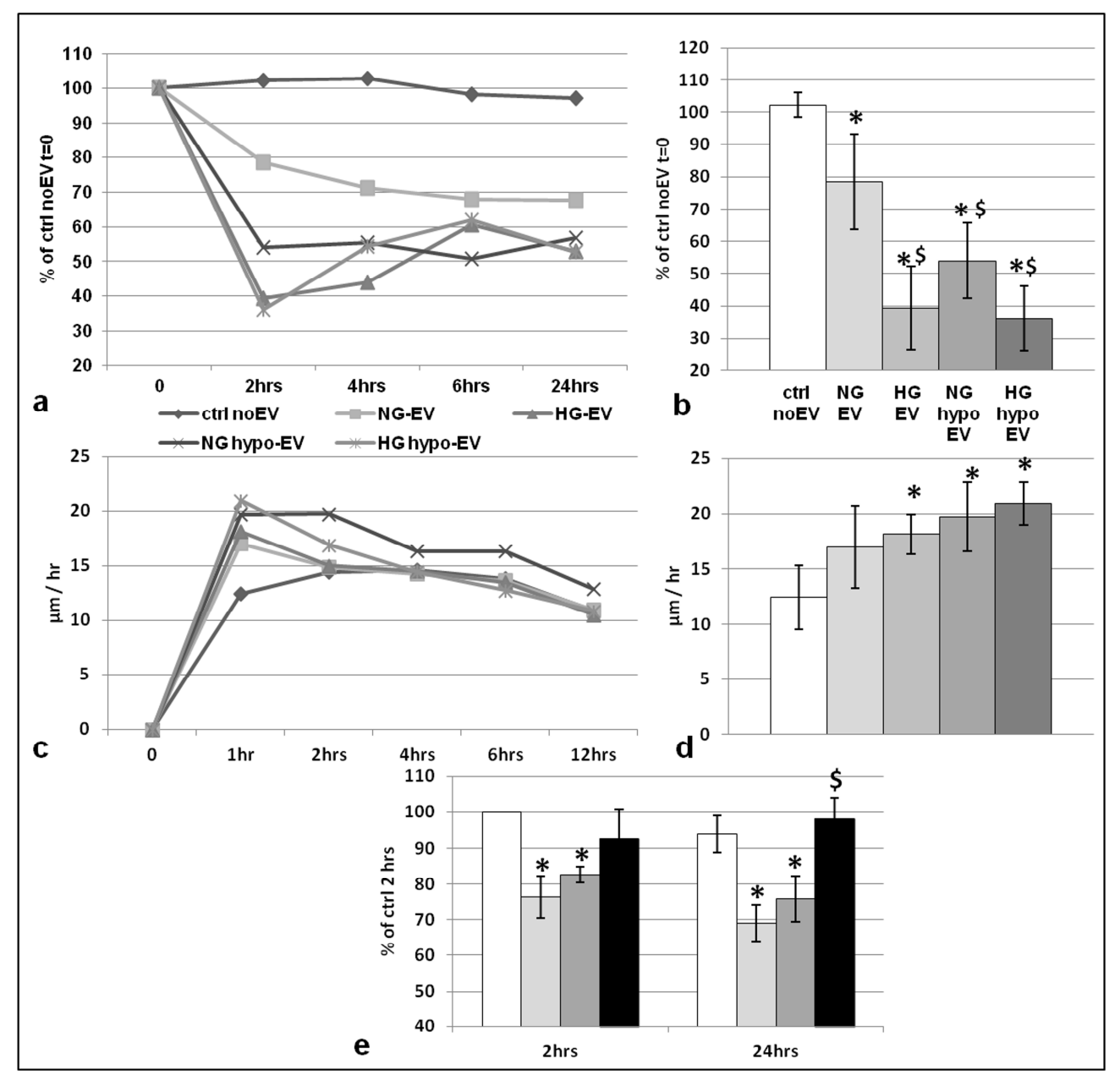

Fig. 2 MSC-derived EV stimulate HRP detachment and migration a) time-course response of HRP to EV stimulation: number of HRP still attached to the well, percentages of control (HRP cultures without EV); b) HRP number after $2 \mathrm{hr} E V$ incubation, percentages of control, ${ }^{*}=p<0.05$ vs control, $\$=p<0.05$ vs cultures treated with EV obtained in physiological conditions (NG-EV); c) HRP migration following EV stimulation, time-course, $\mu \mathrm{m} / \mathrm{hr}$; d) HRP migration after $1 \mathrm{hr}$ EV incubation, ${ }^{*}=p<0.05$ vs control; e) HRP number after 2 and $24 \mathrm{hr}$ incubation with EV derived from bone-marrow MSC (light grey bar), adipose MSC (dark grey), and EC (black), in comparison with untreated control (white). * $p<0.05$ vs control (negative difference), $\$ p<0.05$ vs control (positive difference) 


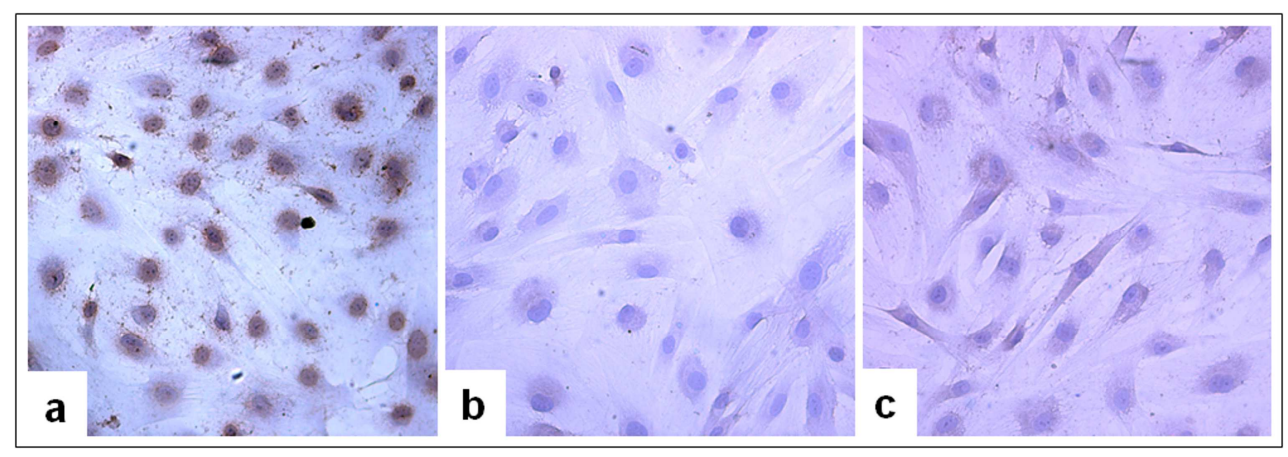

Fig. 3 Viability of detached HRP after EV stimulation (TUNEL assay) a) positive control (HRP treated with DNase); b) negative control (untreated HRP culture); c) HRP detached from cultures following EV stimulation and re-seeded in new wells

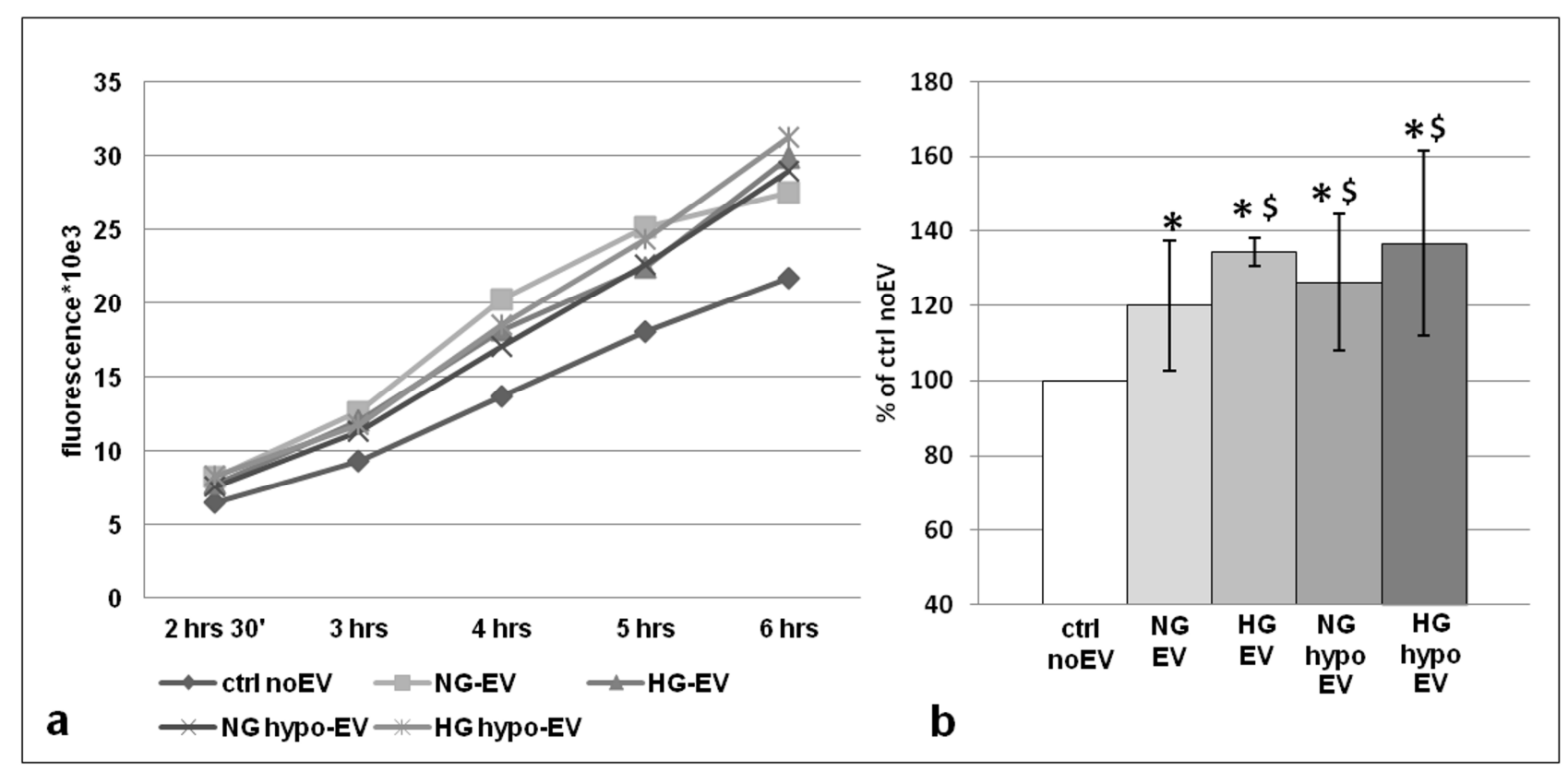

Fig. 4 EV increase blood-barrier permeability a) time-course permeability of EC/HRP co-cultures on transwell inserts, after EV stimulation; b) permeability after $6 \mathrm{hr}$ exposure. ${ }^{*}=p<0.05$ vs control without EV, $\$$ $=p<0.05$ vs cultures treated with NG-EV 


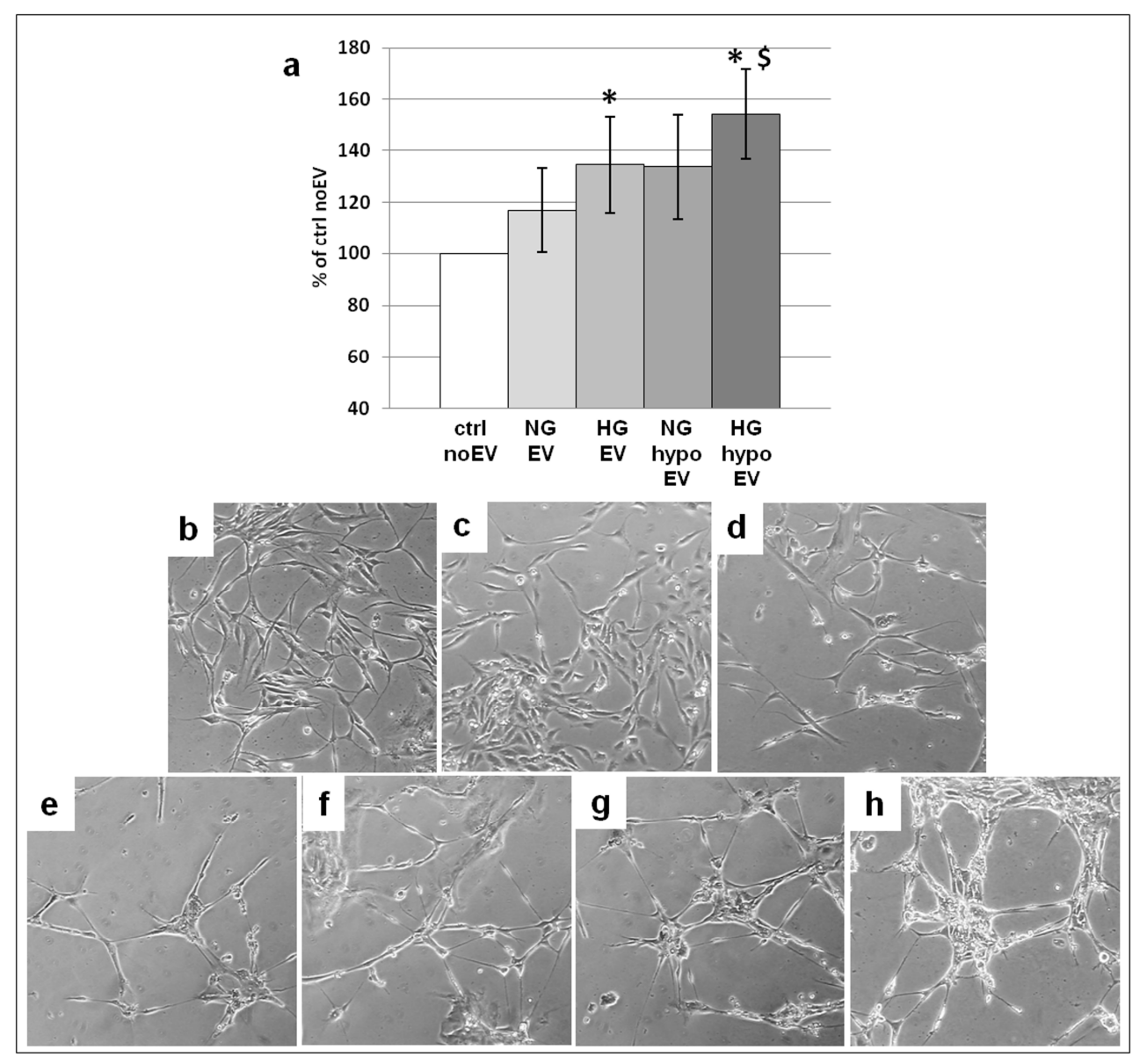

Fig. $5 \mathrm{EV}$ enhance in vitro formation of vessel-like structures by HRP/EC co-cultures a) quantitative analysis of newly-formed vessel-like structures after $48 \mathrm{hr} E V$ incubation, percentages of untreated control. * $p<0.05$ vs control, $\$ p<0.05$ vs HG-EV. b-h) vessel-like structure formation by EC/HRP co-cultures: b) HRP alone; c) EC alone; d) untreated HRP/EC co-cultures (ctrl no EV); e) NG-EV; f) HG-EV, g) NG hypo-EV; h) HG hypo-EV 


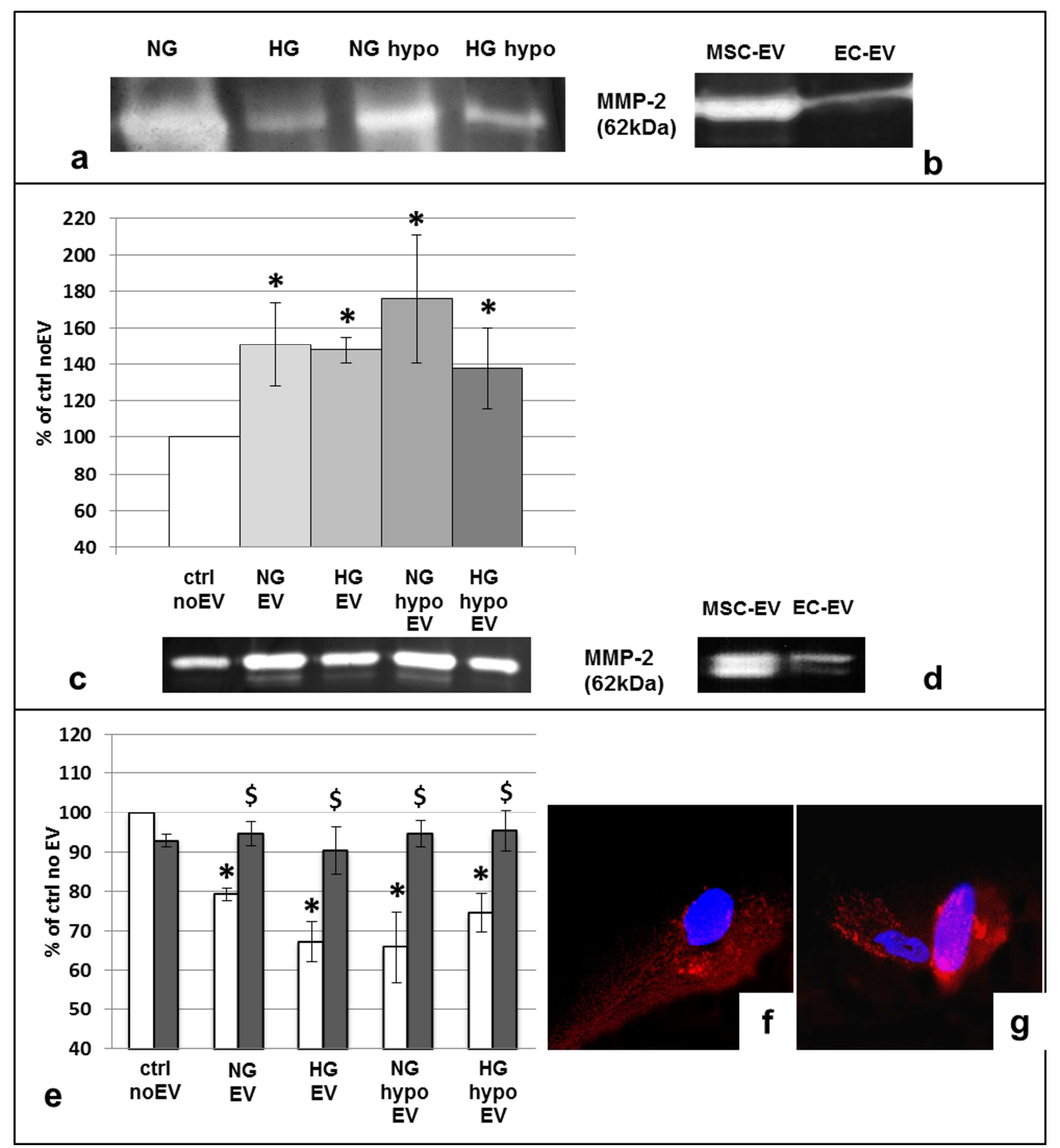

Fig. $6 \mathrm{EV}$ action on HRP is mediated by MMP-2 a) representative zymography of EV showing active MMP2 (62 kDA) expression; b) comparison of MMP-2 content between MSC- and EC-derived EV; c) quantitative analysis of band densities after zymography of HRP supernatants following EV stimulation and image of one representative gel; d) comparative zymography of HRP supernatants following stimulation with MSC- and EC-derived EV; e) HRP number after exposure to NG-EV, HG-EV, NG hypo-EV, HG hypo-EV with (grey bars) or without (white bars) pre-incubation with the MMP inhibitor batimastat, percentages of untreated control ${ }^{*}=p<0.05$ vs ctrl noEV, $\$=p<0.05$ vs correspondent EV without batimastat; $f-g$ ) batimastat does not impede EV internalization into pericytes: HRP incubated for 2 hrs with EV (f) and batimastat pre-treated EV (g) 